\title{
Characterizations of Probability Distributions via Bivariate Regression of Record Values
}

\author{
George P. Yanev M. Ahsanullah M.I. Beg
}

March 7, 2022

\begin{abstract}
Bairamov et al. (2005) characterize the exponential distribution in terms of the regression of a function of a record value with its adjacent record values as covariates. We extend these results to the case of nonadjacent covariates. We also consider a more general setting involving monotone transformations. As special cases, we present characterizations involving weighted arithmetic, geometric, and harmonic means.

Keywords characterization, non-adjacent record values. exponential distribution
\end{abstract}

\section{Introduction and main results}

Let $X_{1}, X_{2}, \ldots$ be independent copies of a random variable $X$ whose distribution function is denoted by $F$. There is a number of studies on characterizations of $F$ by means of regression relations of a function of one record value on one or two other record values. For a recent paper on the subject we refer to Pakes (2004) (see also Ahsanullah and Raqab (2006), Chapter 6). Denote upper record times by $L(1)=1$ and, for $n>1$,

$$
L(n)=\min \left\{j: j>L(n-1) \text { and } X_{j}>X_{L(n-1)}\right\},
$$

and the corresponding upper record value by $X(n)=X_{L(n)}$; see Nevzorov (2001). Gupta and Ahsanullah (2004) study the characterization of $F$ by means of the equation

$$
E[\psi(X(n)) \mid X(n-k)=z]=\varphi(z),
$$

for $k=1$ and $k=2$, where the functions $\psi$ and $\varphi$ satisfy certain regularity conditions. Bairamov et al. (2005) consider a characterization of the exponential distribution in terms of the regression on two adjacent record values

$$
E[\psi(X(n)) \mid X(n-1)=u, X(n+1)=v] \quad\left(l_{F}<u<v<r_{F}\right),
$$

where $l_{F}=\inf \{x: F(x)>0\}$ and $r_{F}=\sup \{x: F(x)<1\}$ are the left and right extremities of $F$, respectively. 
The aim of this paper is to extend the results given in Bairamov et al. (2005) by studying characterizations of $F$ in terms of the regression on two non-adjacent record values

$$
E[\psi(X(n)) \mid X(n-k)=u, X(n+r)=v] \quad\left(l_{F}<u<v<r_{F}\right),
$$

where $1 \leq k \leq n-1$ and $r \geq 1$. Further on, for a given function $h$, we adopt the notation

$$
M(u, v)=\frac{h(v)-h(u)}{v-u},{ }_{i} M_{j}(u, v)=\frac{\partial^{i+j}}{\partial u^{i} \partial v^{j}}\left(\frac{h(v)-h(u)}{v-u}\right) \quad(u \neq v),
$$

as well as ${ }_{i} M(u, v)$ and $M_{j}(u, v)$ for the $i$ th and $j$ th partial derivative of $M(u, v)$ with respect to $u$ and $v$, respectively. Bairamov et al. (2005) characterize the exponential distribution as follows.

Theorem (Bairamov et al. (2005)). Suppose $F$ is absolutely continuous with density $f$, that $h$ is continuous in $\left[l_{F}, r_{F}\right]$ and continuously differentiable in $\left(l_{F}, r_{F}\right)$, and that almost everywhere in this open interval

$$
h^{\prime}(x) \neq M\left(l_{F}, x\right) .
$$

Then

$$
E\left[h^{\prime}(X(n)) \mid X(n-1)=u, X(n+1)=v\right]=M(u, v) \quad\left(l_{F}<u<v<r_{F}\right)
$$

holds if and only if $l_{F}>-\infty, r_{F}=\infty$ and

$$
F(x)=1-e^{-c\left(x-l_{F}\right)} \quad\left(x \geq l_{F}\right),
$$

where $c>0$ is an arbitrary constant.

Next result is a generalization of the above theorem to the case of regression on a pair of non-adjacent record values. Namely, (3) is extended for $1 \leq k \leq n-1$ and $r \geq 1$ to

$$
\begin{aligned}
& E\left[h^{(k+r-1)}(X(n)) \mid X(n-k)=u, X(n+r)=v\right] \\
& \quad=\frac{(k+r-1) !}{(k-1) !(r-1) !}{ }^{r-1} M_{k-1}(u, v) \quad\left(l_{F}<u<v<r_{F}\right) .
\end{aligned}
$$

We consider two cases with respect to the spacings from the right record value in the condition as follows: $X(n)$ is one spacing away (there is a gap of size one); or $X(n)$ is two or more spacings away (there is a gap of size two or more). The techniques of the proofs in these two cases differ. 
Theorem 1. Suppose $F$ is absolutely continuous and $h$ is continuous in $\left[l_{F}, r_{F}\right]$ and $h^{(k+r-1)}(x)$ is continuous in $\left(l_{F}, r_{F}\right)$ for $1 \leq k \leq n-1$ and $r \geq 1$.

A. Let $r=1,1 \leq k \leq n-1$ and

$$
M_{k}\left(l_{F}, v\right) \neq 0 \quad\left(l_{F}<v<r_{F}\right) .
$$

Then (4) holds if and only if

$$
F(x)=1-e^{-c\left(x-l_{F}\right)} \quad\left(x \geq l_{F}\right) \quad \text { and } \quad l_{F}>-\infty, r_{F}=\infty,
$$

where $c>0$ is an arbitrary constant.

B. Let $r \geq 2,2 \leq k \leq n-1$ and ${ }_{r} M_{k-1}\left(l_{F}, v\right) \neq 0\left(l_{F}<v<r_{F}\right)$. Then both (4) and

$$
\begin{aligned}
& E\left[h^{(k+r-1)}(X(n)) \mid X(n-k+1)=u_{2}, X(n+r)=v\right] \\
& \quad=\frac{(k+r-2) !}{(k-2) !(r-1) !} r-1 M_{k-2}^{\prime}\left(u_{2}, v\right) \quad\left(l_{F}<u<u_{2}<v<r_{F}\right),
\end{aligned}
$$

where $M^{\prime}(u, v)=\left[h^{\prime}(v)-h^{\prime}(u)\right] /(v-u)$, hold if and only if (6) is true.

Remarks. (i) If $k=r=1$, then Theorem $1 \mathrm{~A}$ coincides with Theorem 1 in Bairamov et al. (2005) because, using (9) below, the assumption (2) can be written as $M_{1}\left(l_{F}, v\right) \neq 0$. (ii) The statement in Theorem $1 \mathrm{~A}$ holds true when $k=1$ and $r \geq 1$ as well (with ${ }_{r} M\left(l_{F}, v\right) \neq 0$ instead of (5)) and can be proved along the same lines, differentiating with respect to $u$ instead of $v$.

The following result is an extension of Theorem 2 in Bairamov et al. (2005) to regression on a pair of non-adjacent covariates. As we will see in the next section, it follows from Theorem 1 choosing $h(x)=x^{k+r} /(k+r)$ !.

Theorem 2. A. Let $r=1$. For $1 \leq k \leq n-1$

$$
E[X(n) \mid X(n-k)=u, X(n+r)=v]=\frac{r u+k v}{k+1} \quad\left(l_{F}<u<v<r_{F}\right)
$$

if and only if the continuous r.v. $X$ has the exponential distribution (6).

B. If $r \geq 2$ and $2 \leq k \leq n-1$, then both (8) and for $l_{F}<u<u_{2}<v<r_{F}$

$$
E\left[X(n) \mid X(n-k+1)=u_{2}, X(n+r)=v\right]=\frac{r u_{2}+(k-1) v}{r+k-1}
$$

hold if and only if the continuous r.v. $X$ has the exponential distribution (6).

The proofs of Theorems 1 and 2 are given in Section 2. In Section 3 we consider monotone transformations which extend the results in the previous sections to a more general setting. Illustrations are given in terms of characterizations involving arithmetic, geometric, and harmonic means.

\section{Proofs of Theorems 1 and 2}

Further on we will need some recurrent relations for the derivatives of $M(u, v)$ given in the following lemma. 
Lemma 1. Let $h(x)$ be a given function and for integer $i, j \geq 1$ define $M(u, v),{ }_{i} M(u, v), M_{j}(u, v)$, and ${ }_{i} M_{j}(u, v)$ as in (11). If $h(x)$ has a continuous derivative of order $\max \{i, j\}$ over the interval $(a, b)$, then for $a<u<v<b$

$$
M_{j}(u, v)=\frac{h^{(j)}(v)-j M_{j-1}(u, v)}{v-u}, \quad{ }_{j} M(u, v)=\frac{j_{j-1} M(u, v)-h^{(j)}(u)}{v-u}
$$

and

$$
{ }_{i} M_{j}(u, v)=\frac{i_{i-1} M_{j}(u, v)-j_{i} M_{j-1}(u, v)}{v-u},
$$

where $M_{1}(u, v)$ and ${ }_{1} M(u, v)$ are given in (9) and ${ }_{1} M_{1}(u, v)=\left(M_{1}(u, v)-\right.$ $\left.{ }_{1} M(u, v)\right) /(v-u)$.

Proof. It is not difficult to prove (9) by induction. We will proceed with the proof of (10). One can check (10) for $i=1,2$ and $j=1,2$. Assume that (10) holds for some $(i, j)$. Fixing $i$ we shall prove it for $(i, j+1)$, i.e.,

$$
{ }_{i-1} M_{j+1}(u, v)-(j+1){ }_{i} M_{j}(u, v)=(v-u){ }_{i} M_{j+1}(u, v)
$$

Indeed, using the induction assumption, we have

$$
\begin{aligned}
i_{i-1} & M_{j+1}(u, v)-(j+1){ }_{i} M_{j}(u, v) \\
& =\frac{\partial}{\partial v}\left[i_{i-1} M_{j}(u, v)-j{ }_{i} M_{j-1}(u, v)\right]-{ }_{i} M_{j}(u, v) \\
& =\frac{\partial}{\partial v}\left[(v-u){ }_{i} M_{j}(u, v)\right]-{ }_{i} M_{j}(u, v) \\
& =(v-u){ }_{i} M_{j+1}(u, v) .
\end{aligned}
$$

Similarly, assuming (10) for an $i$ and fixed but arbitrary $j$, one can prove that it holds for $(i+1, j)$. The lemma is proved.

Denote $R(x)=-\ln (1-F(x))$. Using the Markov dependence of record values, one can show (e.g., Ahsanullah (2004)) that the conditional density of $X(n)$ given $X(n-k)=u(1 \leq k \leq n-1)$ and $X(n+r)=v(r \geq 1)$ is

$$
\frac{(k+r-1) !}{(k-1) !(r-1) !}\left[\frac{R(t)-R(u)}{R(v)-R(u)}\right]^{k-1}\left[\frac{R(v)-R(t)}{R(v)-R(u)}\right]^{r-1} \frac{R^{\prime}(t)}{R(v)-R(u)},
$$

where $u<t<v$.

We will need the following lemma, which is of independent interest as well.

Lemma 2. Let $h(x)$ be a continuous in $\left[l_{F}, r_{F}\right]$ function such that $h^{(k+r-1)}(x)$ is continuous in $\left(l_{F}, r_{F}\right)$ for $1 \leq k \leq n-1$ and $r \geq 1$. If

$$
F(x)=1-e^{-c\left(x-l_{F}\right)} \quad\left(x \geq l_{F}\right) \quad \text { and } \quad l_{F}>-\infty, r_{F}=\infty,
$$

where $c>0$ is an arbitrary constant, then

$$
\begin{aligned}
& E\left[h^{(k+r-1)}(X(n)) \mid X(n-k)=u, X(n+r)=v\right] \\
& \quad=\frac{(k+r-1) !}{(k-1) !(r-1) !}{ }^{r-1} M_{k-1}(u, v) \quad\left(l_{F}<u<v<r_{F}\right) .
\end{aligned}
$$


Proof. It is not difficult to verify (12) for $k=r=1$. Let us prove it for $r=1$ and any $1 \leq k \leq n-1$, i.e.,

$$
E\left[h^{(k)}(X(n)) \mid X(n-k)=u, X(n+1)=v\right]=k M_{k-1}(u, v)
$$

for $1 \leq k \leq n-1$. Assuming that (13) is true for $k=i \quad(1 \leq i \leq n-1)$, we will prove it for $k=i+1$. Indeed, making use of (11) with $R(x)=c\left(x-l_{F}\right)$ and the induction assumption, we obtain

$$
\begin{aligned}
E & {\left[h^{(i+1)}(X(n)) \mid X(n-i-1)=u, X(n+1)=v\right] } \\
& =\frac{i+1}{(v-u)^{i+1}} \int_{u}^{v} h^{(i+1)}(t)(t-u)^{i} d t \\
& =\frac{i+1}{(v-u)^{i+1}}\left[h^{(i)}(v)(v-u)^{i}-i \int_{u}^{v} h^{(i)}(t)(t-u)^{i-1} d t\right] \\
& =\frac{i+1}{(v-u)^{i+1}}\left[h^{(i)}(v)(v-u)^{i}-(v-u)^{i} E\left[h^{(i)}(X(n)) \mid X(n-i)=u, X(n+1)=v\right]\right] \\
& =\frac{i+1}{(v-u)^{i+1}}\left[h^{(i)}(v)(v-u)^{i}-i(v-u)^{i} M_{i-1}(u, v)\right] \\
& =\frac{i+1}{v-u}\left[h^{(i)}(v)-i M_{i-1}(u, v)\right] \\
& =(i+1) M_{i}(u, v),
\end{aligned}
$$

where the last equality follows from (9). This proves (13) for $k=i+1$ and thus (12) is true for $r=1$ and any $1 \leq k \leq n-1$. Similarly one can prove (12) for $k=1$ and any $r \geq 1$, i.e.,

$$
E\left[h^{(r)}(X(n)) \mid X(n-1)=u, X(n+r)=v\right]=r_{r-1} M(u, v)
$$

To complete the proof of the lemma we need to show (12) for $r \geq 2$ and $2 \leq k \leq n-1$. Let us assume (12) for $r=j$ and any $2 \leq k \leq n-1$. We will prove it for $r=j+1$ and any $2 \leq k \leq n-1$. Since the left-hand side of (12) for $r=j+1$ is

$$
\begin{aligned}
E & {\left[h^{(k+j)}(X(n)) \mid X(n-k)=u, X(n+j+1)=v\right] } \\
& =\frac{(k+j) !(v-u)^{-(k+j)}}{(k-1) ! j !} \int_{u}^{v} h^{(k+j)}(t)(t-u)^{k-1}(v-t)^{r} d t,
\end{aligned}
$$

to prove (12) for $r=j+1$ we need to show that for $2 \leq k \leq n-1$

$$
\begin{aligned}
I(k, j+1) & =\int_{u}^{v} h^{(k+j)}(t)(t-u)^{k-1}(v-t)^{j} d t \\
& =(v-u)^{k+j}{ }_{j} M_{k-1}(u, v)
\end{aligned}
$$

under the induction assumption that for any $2 \leq k \leq n-1$

$$
\begin{aligned}
I(k, j) & =\int_{u}^{v} h^{(k+j-1)}(t)(t-u)^{k-1}(v-t)^{j-1} d t \\
& =(v-u)^{k+j-1}{ }_{j-1} M_{k-1}(u, v)
\end{aligned}
$$


Integrating by parts, we have for $2 \leq k \leq n-1$

$$
\begin{aligned}
I(k, j)= & \int_{u}^{v} h^{(k+j-1)}(t)(t-u)^{k-1}(v-t)^{j-1} d t \\
= & \frac{1}{j} \int_{u}^{v} h^{(k+j)}(t)(t-u)^{k-1}(v-t)^{j} d t \\
& +\frac{k-1}{j} \int_{u}^{v} h^{(k+j-1)}(t)(t-u)^{k-1}(v-t)^{j} d t
\end{aligned}
$$

Let $(x)_{n}$ be the falling factorial, i.e., $(x)_{n}=x(x-1) \ldots(x-n+1)(n \geq 1)$ and $(x)_{0}=1$. After iterating, we have for $2 \leq k \leq n-1$

$$
\begin{aligned}
& I(k, j+1) \\
& \quad=j I(k, j)-(k-1) I(k-1, j+1) \\
& \quad=j \sum_{i=0}^{k-2}(-1)^{i}(k-1)_{(i)} I(k-i, j)+(-1)^{k-1}(k-1)_{(k-1)} \int_{u}^{v} h^{(j+1)}(t)(v-t)^{j} d t
\end{aligned}
$$

Observe that (14) and (9) lead to

$$
\begin{aligned}
\int_{u}^{v} & h^{(j+1)}(t)(v-t)^{j} d t \\
& =-h^{(j)}(u)(v-u)^{j}+j \int_{u}^{v} h^{(j)}(t)(v-t)^{j-1} d t \\
& =(v-u)^{j}\left\{-h^{(j)}(u)+E\left[h^{(j)}(X(n)) \mid X(n-1)=u, X(n+j)=v\right]\right\} \\
& =(v-u)^{j}\left[-h^{(j)}(u)+j{ }_{j-1} M(u, v)\right] \\
& =(v-u)^{j}\left[-h^{(j)}(u)+(v-u)_{j} M(u, v)+h^{(j)}(u)\right] \\
& =(v-u)^{j+1}{ }_{j} M(u, v)
\end{aligned}
$$

Using the induction assumption (16) and (18) we write (17) as

$$
\begin{aligned}
\frac{I(k, j+1)}{(v-u)^{j+1}}= & j \sum_{i=0}^{k-2}(-1)^{i}(k-1)_{(i)}(v-u)^{k-2-i}{ }_{j-1} M_{k-1-i}(u, v) \\
& -(-1)^{k-2}(k-1)_{(k-1)} j M(u, v)
\end{aligned}
$$

Now, applying (10) and iterating, we obtain

$$
\begin{aligned}
& \frac{I(k, j+1)}{(v-u)^{j+1}} \\
& \quad=j \sum_{i=0}^{k-3}(-1)^{i}(k-1)_{(i)}(v-u)^{k-2-i}{ }_{j-1} M_{k-1-i}(u, v) \\
& \quad+(-1)^{k-2}(k-1)_{(k-2)}\left[j_{j-1} M_{1}(u, v)-{ }_{j} M(u, v)\right]
\end{aligned}
$$




$$
\begin{aligned}
= & j \sum_{i=0}^{k-3}(-1)^{i}(k-1)_{(i)}(v-u)^{k-2-i}{ }_{j-1} M_{k-1-i}(u, v) \\
& \quad-(-1)^{k-3}(k-1)_{(k-2)}(v-u)_{j} M_{1}(u, v) \\
= & j \sum_{i=0}^{k-4}(-1)^{i}(k-1)_{(i)}(v-u)^{k-2-i}{ }_{j-1} M_{k-1-i}(u, v) \\
& \quad+(-1)^{k-3}(k-1)_{(k-3)}(v-u)\left[j_{j-1} M_{2}(u, v)-2{ }_{j} M_{1}(u, v)\right] \\
= & j \sum_{i=0}^{k-4}(-1)^{i}(k-1)_{(i)}(v-u)^{k-2-i}{ }_{j-1} M_{k-1-i}(u, v) \\
& \quad \cdots \quad-(-1)^{k-4}(k-1)_{(k-3)}(v-u)^{2}{ }_{j-1} M_{2}(u, v) \\
= & j(v-u)^{k-2}{ }_{j-1} M_{k-1}-(k-1)(v-u)^{k-2}{ }_{j-1} M_{k-2}(u, v) \\
= & (v-u)^{k-1}{ }_{j-1} M_{k-1}(u, v) .
\end{aligned}
$$

This implies (15). Similarly assuming (12) for $k=i(2 \leq i \leq n-2)$ and any $r \geq 2$ one can prove it for $k=i+1$ and $r \geq 2$. The lemma is proved.

\subsection{Proof of Theorem 1A}

Assume (41). Setting $r=1$ in (11), we obtain from (4)

$$
M_{k-1}(u, v)[R(v)-R(u)]^{k}=\int_{u}^{v} h^{(k)}(t)[R(t)-R(u)]^{k-1} R^{\prime}(t) d t .
$$

Letting $u \rightarrow l_{F}^{+}$and noting that the integrand is continuous and $\lim _{u \rightarrow l_{F}^{+}} R(u)=$ $\lim _{u \rightarrow l_{F}^{+}}(-\ln (1-F(u)))=0$ we simplify to

$$
M_{k-1}\left(l_{F}, v\right)[R(v)]^{k}=\int_{l_{F}}^{v} h^{(k)}(t)[R(t)]^{k-1} R^{\prime}(t) d t .
$$

Differentiating both sides of the above equation with respect to $v$, we obtain

$$
k M_{k-1}\left(l_{F}, v\right)[R(v)]^{k-1} R^{\prime}(v)+M_{k}\left(l_{F}, v\right)[R(v)]^{k}=h^{(k)}(v)[R(v)]^{k-1} R^{\prime}(v)
$$

Rearranging and taking into account (9),

$$
\begin{aligned}
\frac{R^{\prime}(v)}{R(v)} & =\frac{M_{k}\left(l_{F}, v\right)}{h^{(k)}(v)-k M_{k-1}\left(l_{F}, v\right)} \\
& =\frac{M_{k}\left(l_{F}, v\right)}{\left(v-l_{F}\right) M_{k}\left(l_{F}, v\right)} \\
& =\frac{1}{v-l_{F}}
\end{aligned}
$$

(provided that $M_{k}\left(l_{F}, v\right) \neq 0$ ) and hence (6) holds. It follows that $l_{F}>-\infty$ and $c>0$, and the continuity of $F$ implies that $r_{F}=\infty$.

The converse statement follows from Lemma 2. The proof is complete. 


\subsection{Proof of Theorem 1B}

Assume both (4) and (7) are true. Formula (11) together with (4) imply

$$
\begin{aligned}
{ }_{r-1} & M_{k-1}(u, v)[R(v)-R(u)]^{k+r-1} \\
= & \int_{u}^{v} h^{(k+r-1)}(t)[R(v)-R(t)]^{r-1}[R(t)-R(u)]^{k-1} R^{\prime}(t) d t .
\end{aligned}
$$

Since the integrand is continuous, differentiating both sides of the above equation with respect to $u$, we obtain

$$
\begin{aligned}
{ }_{r} M_{k-1} & (u, v)[R(v)-R(u)]^{k+r-1} \\
& -(k+r-1)[R(v)-R(u)]^{k+r-2} R^{\prime}(u){ }_{r-1} M_{k-1}(u, v) \\
& =-(k-1) R^{\prime}(u) \int_{u}^{v} h^{(k+r-1)}(t)[R(v)-R(t)]^{r-1}[R(t)-R(u)]^{k-2} R^{\prime}(t) d t .
\end{aligned}
$$

On the other hand, (7) and (11) lead to

$$
\begin{aligned}
& { }_{r-1} M_{k-2}^{\prime}\left(u_{2}, v\right)\left[R(v)-R\left(u_{2}\right)\right]^{k+r-2} \\
= & \int_{u_{2}}^{v} h^{(k+r-1)}(t)[R(v)-R(t)]^{r-1}\left[R(t)-R\left(u_{2}\right)\right]^{k-2} R^{\prime}(t) d t .
\end{aligned}
$$

Therefore, letting $u_{2} \rightarrow u^{+}$in (21) and rearranging terms, we write (20) as

$$
\frac{R^{\prime}(u)}{R(u)-R(v)}=\frac{{ }_{r} M_{k-1}(u, v)}{(k-1)_{r-1} M_{k-2}^{\prime}(u, v)-(k+r-1)_{r-1} M_{k-1}(u, v)}
$$

provided that the denominator in the right-hand side is not 0 . (This is equivalent to ${ }_{r} M_{k-1}(u, v) \neq 0$, as we will see below.) Since

$$
\begin{aligned}
{ }_{r-1} M_{k-2}^{\prime}(u, v) & =\frac{\partial^{k+r-3}}{\partial u^{r-1} \partial v^{k-2}}\left[\frac{h^{\prime}(v)-h^{\prime}(u)}{v-u}\right] \\
& =\frac{\partial^{k+r-3}}{\partial u^{r-1} \partial v^{k-2}}\left[M_{1}(u, v)+{ }_{1} M(u, v)\right] \\
& ={ }_{r-1} M_{k-1}(u, v)+{ }_{r} M_{k-2}(u, v),
\end{aligned}
$$

for the denominator in (22) we have

$$
\begin{aligned}
& (k-1)_{r-1} M_{k-2}^{\prime}(u, v)-(k+r-1)_{r-1} M_{k-1}(u, v) \\
& =(k-1)\left[{ }_{r-1} M_{k-1}(u, v)+{ }_{r} M_{k-2}(u, v)\right]-(k+r-1)_{r-1} M_{k-1}(u, v) \\
& =(k-1){ }_{r} M_{k-2}(u, v)-r_{r-1} M_{k-1}(u, v) .
\end{aligned}
$$

Finally, from (22)-(23) and applying (10), we obtain

$$
\begin{aligned}
\frac{R^{\prime}(u)}{R(u)-R(v)} & =\frac{{ }_{r} M_{k-1}(u, v)}{(k-1)_{r} M_{k-2}(u, v)-r_{r-1} M_{k-1}(u, v)} \\
& =\frac{{ }_{r} M_{k-1}(u, v)}{{ }_{r} M_{k-1}(u, v)(u-v)} \\
& =\frac{1}{u-v} .
\end{aligned}
$$


Integrating both sides with respect to $u$ from $l_{F}$ to $v$, we obtain

$$
\ln \left[R(v)-R\left(l_{F}\right)\right]=\ln \left(v-l_{F}\right)+\ln c \quad(c>0)
$$

and thus $R(v)=c\left(v-l_{F}\right)$ and (6) follows.

The converse statement in the theorem follows from Lemma 2.

\subsection{Proof of Theorem 2}

Let $h(x)=x^{k+r} /(k+r)$ ! and thus $h^{(k+r-1)}(x)=x$. We shall prove that, with this choice of $h$, (4) becomes

$$
E[X(n) \mid X(n-k)=u, X(n+r)=v]=\frac{r u+k v}{k+r} \quad\left(l_{F}<u<v<r_{F}\right) .
$$

Indeed,

$$
\begin{aligned}
M(u, v) & =\frac{1}{(k+r) !} \frac{v^{k+r}-u^{k+r}}{v-u} \\
& =\frac{v^{k+r-1}+\ldots+v^{k} u^{r-1}+v^{k-1} u^{r}+\ldots+u^{k+r-1}}{(k+r) !}
\end{aligned}
$$

and differentiating $r-1$ and $k-1$ times with respect to $u$ and $v$, we obtain

$$
\begin{aligned}
\frac{(k+r-1) !}{(k-1) !(r-1) !}{ }_{r-1} & M_{k-1}(u, v) \\
& =\frac{(k+r-1) !}{(r-1) !(k-1) !} \frac{(r-1) ! k ! v+r !(k-1) ! u}{(k+r) !} \\
& =\frac{r u+k v}{k+r}
\end{aligned}
$$

which proves (24). Now, if $r=1$ (note that $M_{k}\left(l_{F}, v\right)=l_{F} /(k+1) \neq 0$ ) the claim in Theorem $2 \mathrm{~A}$ follows from Theorem 1A. Similarly to 25 one can see that (7) becomes

$$
\begin{aligned}
E[X(n) \mid X(n-k+1) & \left.=u_{2}, X(n+r)=v\right] \\
& =\frac{(k+r-2) !}{(k-2) !(r-1) !} r-1 M_{k-2}^{\prime}\left(u_{2}, v\right) \\
& =\frac{r u_{2}+(k-1) v}{k+r-1} .
\end{aligned}
$$

Theorem 1B, (25) and (26) imply Theorem 2B. The proof is complete.

\section{Monotone transformations and some particu- lar cases}

In this section, following Bairamov et al. (2005), we give a formal generalization of Theorem 1, involving a monotone transformation of $X$. Let $Y$ be a random 
variable with distribution function $G$. The corresponding upper record values are denoted by $Y(n)$. The following extension of Theorem $1 \mathrm{~A}$ holds. The proof is similar to that of Theorem 3 in Bairamov et al. (2005) and it is omitted here. Denote for $i, j \geq 0$

$$
{ }_{i} M_{j}(T(s), T(t))=\left.\frac{\partial^{i+j}}{\partial x^{i} \partial y^{j}}\left(\frac{h(y)-h(x)}{y-x}\right)\right|_{x=T(s), y=T(t)} \quad(y \neq x) .
$$

Theorem 3. Suppose that:

(i) $Y$ has a continuous distribution function $G$ on $\left[l_{G}, r_{G}\right]$;

(ii) the function $T$ is continuous and strictly increasing in $\left(l_{G}, r_{G}\right), \tau=$ $T\left(l_{G+}\right)>-\infty$ and $T\left(r_{G}\right)=\infty$; and

(iii) $h^{(k+r-1)}(x)$ is continuous in $(\tau, \infty)$ for $1 \leq k \leq n-1$ and $r \geq 1$.

A. Let $r=1$ and $M_{k}(\tau, T(t)) \neq 0\left(l_{G}<t<r_{G}\right)$. Then for $1 \leq k \leq n-1$ and $l_{G}<s<t<r_{G}$

$$
E\left[h^{(k)}(T(Y(n))) \mid Y(n-k)=s, Y(n+1)=t\right]=k M_{k-1}(T(s), T(t))
$$

if and only if

$$
G(y)=1-e^{-c[T(y)-\tau]} \quad\left(l_{G}<y<r_{G}\right)
$$

where $c>0$ is an arbitrary constant.

B. Let $r \geq 2$ and $2 \leq k \leq n-1$, and ${ }_{r} M_{k-1}(\tau, T(t)) \neq 0$ where $l_{G}<t<r_{G}$. Then both (27) and for $l_{G}<s<s_{2}<t<r_{G}$

$$
\begin{gathered}
E\left[h^{(k+r-1)}(T(Y(n))) \mid Y(n-k+1)=s_{2}, Y(n+r)=t\right] \\
=\frac{(k+r-2) !}{(k-2) !(r-1) !}{ }_{r-1} M_{k-2}^{\prime}\left(T\left(s_{2}\right), T(t)\right)
\end{gathered}
$$

hold if and only if (28) is true.

Remark. An analog of Theorem 3 when $T$ is a strictly decreasing function holds as well; for the case $k=r=1$ see Bairamov et al. (2005), Theorem 3.

Different choices of functions $h$ and $T$ in the above theorem yield many characterization results. The corollary below gives a characterization that involves a weighted arithmetic mean.

Corollary 1. Let (i) and (ii) of Theorem 3 hold. If $1 \leq k \leq n-1$, then for a strictly increasing function $g$

$$
E[g(Y(n)) \mid Y(n-k)=s, Y(n+1)=t]=\frac{k g(t)+g(s)}{k+1} \quad\left(l_{G}<s<t<r_{G}\right)
$$

holds if and only if

$$
G(y)=1-e^{-c[g(y)-g(\tau)]} \quad\left(l_{G}<y<r_{G}\right)
$$

where $c>0$ is an arbitrary constant.

Proof. The corollary follows from Theorem $2 \mathrm{~A}$ and Theorem 3 setting $T(y)=g(y)$ and $h(x)=x^{k+1} /(k+1) !$. 
Next corollary presents characterizations involving a geometric mean as a special case.

Corollary 2. Let (i) and (ii) of Theorem 3 hold. If $1 \leq k \leq n-1$, then for a strictly decreasing function $g$ and $l_{G}<s<t<r_{G}$

$$
E[g(Y(n)) \mid Y(n-k)=s, Y(n+1)=t]=[g(t)]^{k /(k+1)}[g(s)]^{r /(k+1)}
$$

holds if and only if

$$
G(y)=1-e^{-c\left\{[g(y)]^{-1 /(k+1)}-[g(\tau)]^{-1 /(k+1)}\right\}} \quad\left(l_{G}<y<r_{G}\right),
$$

where $c>0$ is an arbitrary constant.

Proof. We will show that if $h(x)=-x^{-1}$, then for $j=1,2, \ldots$

$$
M_{j}(x, y)=(-1)^{j} \frac{j !}{x y^{j+1}} .
$$

Indeed, one can check that $M_{1}(x, y)=-1 /\left(x y^{2}\right)$. Assuming that (30) is true for $j$, we will prove it for $j+1$. Using (9) we have

$$
\begin{aligned}
M_{j+1}(x, y) & =\frac{1}{y-x}\left[(-1)^{j+2} \frac{(j+1) !}{y^{j+2}}-(j+1)(-1)^{j} \frac{j !}{x y^{j+1}}\right] \\
& =\frac{1}{y-x}\left[(-1)^{j+1} \frac{(j+1) !(y-x)}{x y^{j+2}}\right] \\
& =(-1)^{j+1} \frac{(j+1) !}{x y^{j+2}}
\end{aligned}
$$

and thus (30) follows by induction. Now, let us set for $1 \leq k \leq n-1$

$$
h(x)=\frac{(-1)^{k}}{k ! x} \quad \text { and thus } \quad h^{(k)}(x)=\frac{1}{x^{k+1}} .
$$

It is not difficult to see that, with this choice of $h,(27)$ and (30) yield

$$
E\left[\frac{1}{[T(Y(n))]^{k+1}} \mid Y(n-k)=s, Y(n+1)=t\right]=\frac{1}{T(s)[T(t)]^{k}} .
$$

Setting in the last equation $T(x)=[g(x)]^{-1 /(k+1)}$, leads to the statement of the corollary.

It is worth noting that if $k=r=1$, then the right-hand side in (29) is the geometric mean of $g(s)$ and $g(t)$.

Next corollary is a characterization in terms of a harmonic mean.

Corollary 3. Let (i) and (ii) of Theorem 3 hold. For a strictly decreasing function $g$,

$$
E[g(Y(n)) \mid Y(n-1)=s, Y(n+1)=t]=\frac{2 g(s) g(t)}{g(s)+g(t)} \quad\left(l_{G}<s<t<r_{G}\right) .
$$


holds if and only if

$$
G(y)=1-e^{-c\left\{[g(y)]^{-2}-[g(\tau)]^{-2}\right\}} \quad\left(l_{G}<y<r_{G}\right)
$$

where $c>0$ is an arbitrary constant.

Proof. The result follows from Theorem 3 setting $h(x)=2 x^{1 / 2}$ and $T(y)=$ $[g(y)]^{-2}$.

Finally, let us note that Theorem 3 and its corollaries yield many special cases. In particular, one can easily adjust to our more general setting the examples given in Bairamov et al. (2005). We present here only two examples making use of Corollary 2.

Example 1 (Weibull distribution). Let $l_{G}=0, r_{G}=\infty$, and $g(y)=$ $y^{-\alpha(k+1)}$. Then, according to Corollary $2, Y$ has the Weibull distribution with $G(y)=1-\exp \left\{-c y^{\alpha}\right\}$ if and only if for $1 \leq k \leq n-1$

$$
E\left[[Y(n)]^{-\alpha(k+1)} \mid Y(n-k)=s, Y(n+1)=t\right]=t^{-\alpha k} s^{-\alpha},
$$

where $0<s<t<\infty$. In particular, a random variable $\tilde{Y}$ has the Inverse Weibull distribution with $\tilde{G}(y)=\exp \left\{-c y^{1 / 2}\right\}$ if and only if

$$
E[\tilde{Y}(n) \mid \tilde{Y}(n-1)=s, \tilde{Y}(n+1)=t]=\sqrt{s t} .
$$

Example 2 (Pareto distribution). Let $l_{G}=a>0, r_{G}=\infty$, and $g(y)=$ $[\log y]^{-(k+1)}$. Then, $Y$ has the Pareto distribution with $G(y)=1-(a / y)^{c} \quad(y \geq$ a) if and only if for $1 \leq k \leq n-1$,

$$
E\left[[\log Y(n)]^{-(k+1)} \mid Y(n-k)=s, Y(n+1)=t\right]=[\log t]^{-k}[\log s]^{-1},
$$

where $a \leq s<t<\infty$. Note that the regression relation is independent of $a$.

\section{Acknowledgements}

The authors are grateful to the referees for careful reading and constructive suggestions which were helpful in improving substantially the presentation.

\section{References}

Ahsanullah, M. (2004) Record values - theory and applications. Lanham, MD: University Press of America

Ahsanullah, M. and Raqab, M. (2006) Bounds and Characterizations of Record Statistics. Nova Science Publishers, New York.

Bairamov, I., Ahsanullah, M. and Pakes, A. (2005) A Characterization of continuous distributions via regression on pairs of record values. Aust. N.Z. J. Stat. 47:543-547 
Gupta, R.C. and Ahsanullah M. (2004) Some characterization results based on the conditional expectation of a function of non-adjacent order statistic (record value). Ann. Inst. Statist. Math. 721-732

Nevzorov, V.B. (2001) Records: mathematical theory. Providence, RI: American Mathematical Society

Pakes, A.G. (2004) Product integration and characterization of probability laws. J. Appl. Statist. Sci. 13:11-31 